\title{
Influencia de factores epidemiológicos en la elección de la modalidad de tratamiento renal sustitutivo en la consulta de enfermería de enfermedad renal crónica avanzada
}

\author{
Sonia García Estévez, Gema Vinagre Rea, Patricia Arribas Cobo
}

Enfermeras. Hospital Universitario Infanta Leonor. Madrid

\section{Introducción}

El proceso de información y de elección de la modalidad de diálisis en pacientes con insuficiencia renal crónica constituye un derecho del paciente y una etapa clave para el tratamiento de la persona que padece dicha enfermedad. Esta elección influirá posteriormente en el estilo y calidad de vida del paciente así como en el de sus cuidadores ${ }^{1}$.

Según la ley 41/2002, de 14 de noviembre², básica reguladora de autonomía del paciente y de derechos y obligaciones en materia de información y documentación clínica, la elección de la modalidad de tratamiento de diálisis, se esté o no en lista de trasplante, tiene que hacerla el paciente en todos los casos.

El proceso de elección de la modalidad debe, por ley, incluir un consentimiento informado o similar que garantice la información y asimilación del conocimiento necesario por parte del paciente, para que tome una decisión consciente de la modalidad de diálisis que mejor se adecue a su modo de vida. El consentimiento informado debe contener la información básica

$$
\begin{gathered}
\text { Correspondencia: } \\
\text { Sonia García Estévez } \\
\text { Hosp. U. Infanta Leonor } \\
\text { Servicio de Nefrología } \\
\text { Avenida Gran Vía del Este } 80 \\
\text { 28038. Madrid } \\
\text { e-mail: soniaga.e@ @otmail.com }
\end{gathered}
$$

de la enfermedad, consecuencias de la elección de una modalidad u otra y debe personalizarse para el paciente requiriéndose también la información oral y no solo escrita ${ }^{3}$.

La llegada planificada a diálisis facilita la toma de decisiones, al permitir que el paciente reciba en la consulta de de enfermería Enfermedad Renal Crónica Avanzada (ERCA) educación sobre su enfermedad e información sobre las opciones de tratamiento (Hemodiálisis (HD), Diálisis Peritoneal (DP), Trasplante renal (TR), HD Domiciliaria y Tratamiento Conservador), y en este caso tenga la oportunidad de decidir sobre las condiciones del mismo y su grado de aceptación. La no planificación implica condicionamiento y disminución en la libertad de elección ${ }^{4}$. Teniendo en cuenta la evolución del trasplante de donante cadáver y el incremento de los pacientes que precisan Tratamiento Renal Sustitutivo (TRS), la opción del donante vivo se deberá considerar muy especialmente ${ }^{5}$.

El papel de la consulta de enfermería ERCA en el proceso de elección de la modalidad de tratamiento por parte del paciente es fundamental, ya que garantiza este proceso, mediante la prevención, promoción y rehabilitación de la salud de una forma integral, con una participación activa del paciente, buscando el auto-cuidado y la independencia dentro de un abordaje multidisciplinar ${ }^{6}$.

No obstante, hay que tener en cuenta que en la elección de la modalidad terapéutica (MT) de los pacientes con ERCA que inician TRS influyen múltiples factores, 
entre los cuales destacan además de condicionantes médicos, factores epidemiológicos y las características de cada Servicio de Nefrología. Así como la realidad socio-cultural del paciente, por lo tanto, nos planteamos analizar los distintos factores socio-culturales de nuestra población que podían condicionar la elección de la modalidad de tratamiento.

\section{Objetivos}

- Estudiar las características de la población que acude a nuestra consulta ERCA de enfermería de reciente creación, con respecto a la edad, sexo, etiología ERC, nivel cultural, actividad laboral.

- Valorar con cuales de estos parámetros se relaciona la elección de la modalidad terapéutica (HD, DP, Trasplante vivo o tratamiento conservador).

\section{Material y métodos}

Se trata de un estudio observacional descriptivo de los pacientes incidentes en la consulta de ERCA en el tiempo de estudio.

Las variables recogidas fueron: edad, sexo, actividad laboral, vivienda, nivel cultural, procedencia, tiempo en consulta ERCA, etiología ERC, técnica de elección y técnica de inicio.

Algunos de los datos se obtuvieron de la valoración de enfermería que se realiza en la primera visita del paciente a la consulta y otros de las visitas sucesivas. En nuestra unidad trabajamos con la valoración de enfermería según las necesidades de Virginia Henderson, adaptándola al paciente renal.

Todos los pacientes del estudio, incluidos los pacientes que ingresaron de forma aguda, recibieron educación para la salud sobre ERC, dieta, medicación, factores de riesgo cardiovascular, TRS y acceso para diálisis.

Así mismo, se promovió la continuidad de cuidados en los pacientes que fueron derivados al Equipo de Soporte de Atención Domiciliario (ESAD) de nuestra zona, responsables de la atención de los pacientes que optan por Tratamiento Conservador.

\section{Análisis estadístico}

Las variables cualitativas se presentan en sus frecuencias absoluta y relativa. Las variables cuantitativas se muestran con la media y la desviación estándar (DE), mediana y rango intercuartílico (RIQ) para tiempo de espera. Se comprobó mediante el test de KolmogorovSmirnov la normalidad de los datos.

Para evaluar la elección de modalidad terapéutica (HD, DP, Tratamiento Conservador y trasplante) y técnica de comienzo en función de la edad se utilizó el análisis de la varianza de un factor (ANOVA un factor) para datos independientes. Para determinar si existía asociación entre las variables sexo, actividad laboral, comprensión lectura, etiología, condiciones de domicilio con la elección de modalidad y técnica de comienzo mediante la prueba exacta de Fisher.

Una vez realizado el análisis univariado, se construyó un modelo de regresión logística para ambas variables ajustado por aquellas que en el análisis univariado fueron significativas. Con este modelo se determinaron los OR e IC95\% de la asociación entre elección de modalidad y técnica de comienzo con edad, sexo y actividad laboral.

El nivel de significación aceptado en todos los análisis fue de 0,05 siendo corregido por el método de Bonferroni en las comparaciones múltiples. El paquete estadístico usado fue PASW 18.0 (SPSS Inc., USA).

\section{Resultados}

Se recogieron los datos de 46 pacientes; 15 mujeres $(32,6 \%)$ y 31 hombres $(67,4 \%)$ de edad media 64,61 (rango: 28-90 años).

Los datos se recogieron desde abril 2008 hasta diciembre de 2010, según se fueron incorporando a la consulta de enfermería ERCA.

El tiempo mediano de estancia en programa en consulta de enfermería (excluyendo los 15 pacientes agudos) fue de 8 (RIQ: 3,25 - 14,50) meses.

Las etiologías de la ER eran:

- Nefropatía diabética: 17 pacientes.

- Nefropatía Intersticial crónica: 4 pacientes. 
- Glomerulonefritis proliferativa: 2 pacientes.

- Enfermedad renal vascular: 2 pacientes.

- Otras: 11 pacientes.

- No filiadas: 10 pacientes.

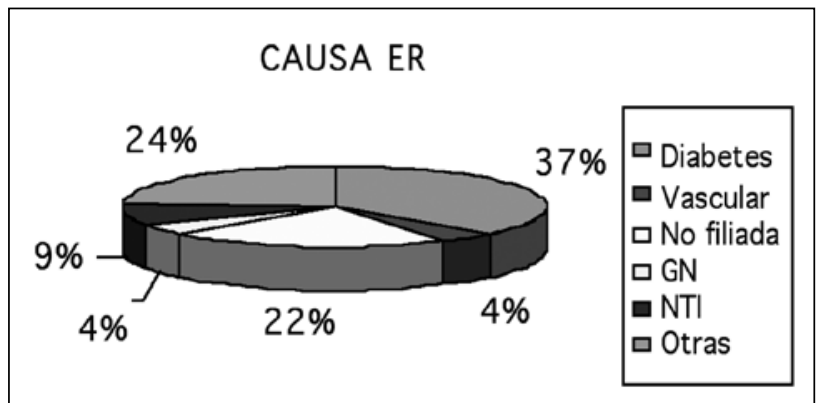

Con respecto a la procedencia de los pacientes estudiados, 24 procedían de consultas externas, 15 fueron pacientes agudos y 7 venían derivados de otros centros.

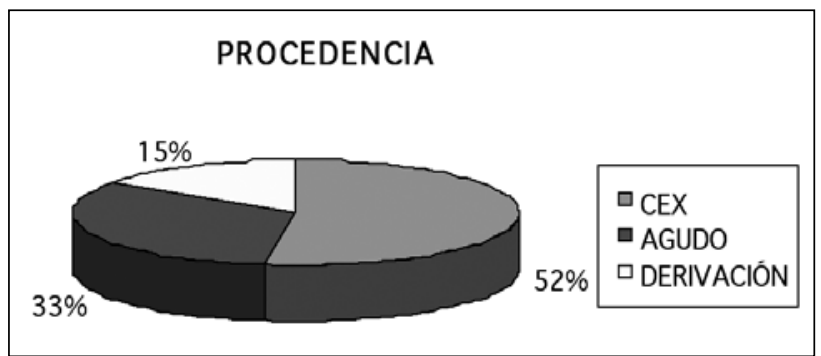

De las variables estudiadas los resultados más relevantes corresponden a la actividad laboral, a la edad, conocimientos lectura-escritura y la técnica elegida. En referencia a la actividad laboral, son significativas las diferencias por sexo, resulta muy llamativo el desempleo en las mujeres, del total de desempleados que suponen el $19,5 \%$, el $77,8 \%$ son mujeres. $p=0,09$.

Tabla 1. Porcentaje de actividad laboral

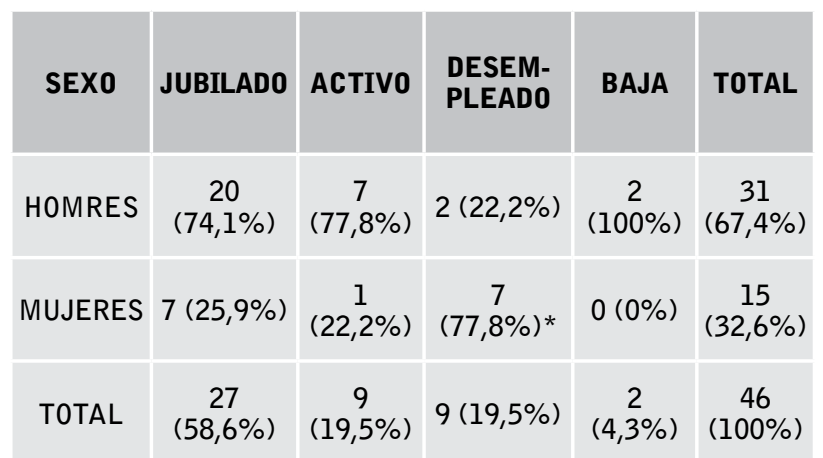

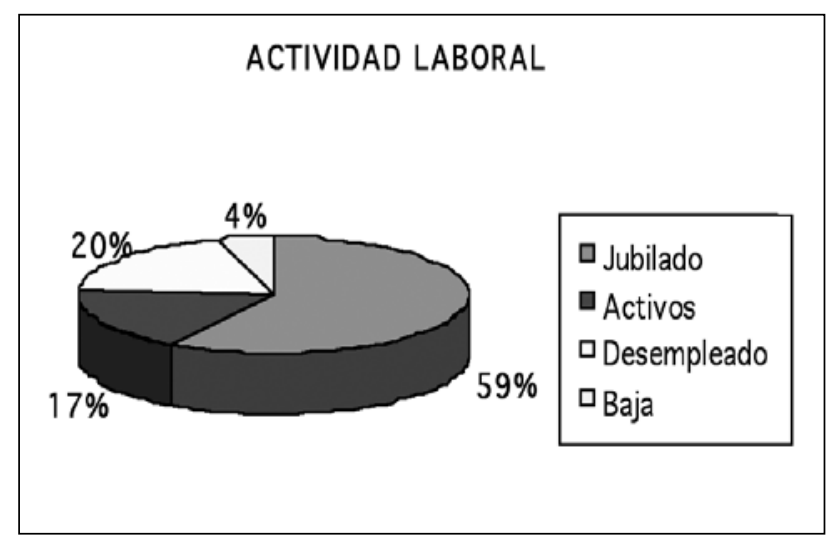

Con respecto a los conocimientos de lectura-escritura relacionada con el sexo, son significativas las diferencias entre sexos en cuanto a saber leer y escribir, hay un $10,8 \%$ del total que no sabe leer ni escribir, de los cuales el $80 \%$ son mujeres $p=0,009$.

Tabla 2. Conocimientos de lectura-escritura relacionada con el sexo

\begin{tabular}{|c|c|c|c|}
\hline SEXO & SI & NO & TOTAL \\
\hline HOMRES & $30(73,2 \%)$ & $1(20 \%)$ & $31(67,4 \%)$ \\
\hline MUJERES & $11(26,8 \%)$ & $4(80 \%)$ & $15(32,6 \%)$ \\
\hline TOTAL & $41(89,2 \%)$ & $5(10,8 \%)$ & $46(100 \%)$ \\
\hline
\end{tabular}

En cuanto a la técnica de elección de los pacientes estudiados, 28 eligieron HD, 9 DP, 7 tratamiento conservador y 2 Trasplante vivo sin diferencias entre sexos.

Posteriormente, la técnica de comienzo de estos pacientes fue: 35 en HD, 4 en DP, 5 en tratamiento conservador y 1 paciente se realizó Trasplante vivo.

La muestra disminuye a 45 pacientes porque de uno de los pacientes que eligió tratamiento conservador no disponemos más datos. 
Tabla 3. Porcentaje de técnica elegida/técnica comienzo

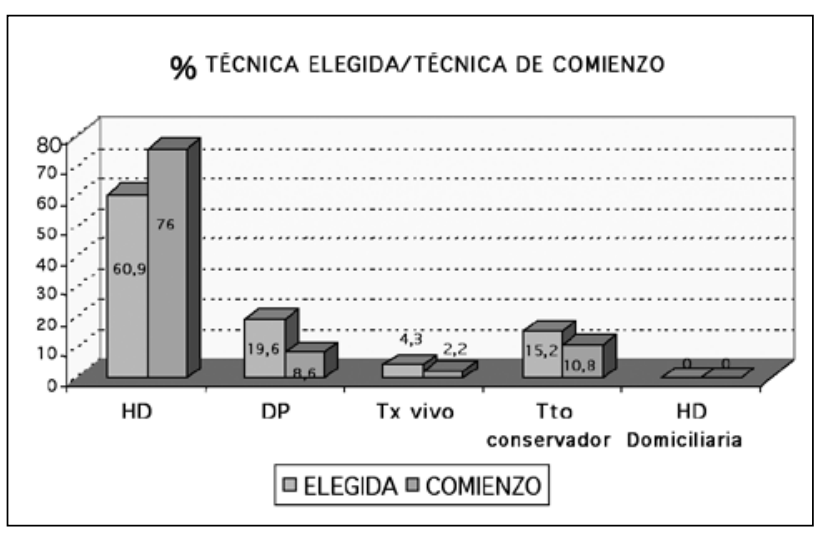

De los pacientes que eligieron DP, $3(33,3 \%)$ tenían contraindicaciones médicas o sociales para la técnica (1 por problemas relacionados con el catéter y 2 por condiciones del domicilio). Otros 2 de los pacientes $(22,2 \%)$ comenzaron primero en HD de forma aguda, posteriormente se les dio información para la elección de tratamiento y eligieron DP.

El $8,7 \%$ del total de los pacientes estudiados no disponía de habitación propia en su vivienda.

Al analizar la edad respecto a la elección del tratamiento por parte del paciente, se han encontrado diferencias estadísticamente significativas $(p<0,001)$. Estas diferencias se han encontrado entre HD vs DP $(p=0.001)$ con una diferencia de medias de 19,8 años (IC95\%: 6,4-33,3) mayor para HD.

También se ha mostrado esta diferencia entre DP vs Tratamiento Conservador $(p<0,001)$ y trasplante vivo vs Tratamiento Conservador $(p=0,002)$.

En el caso de Técnica elegida, la $n=45$ al no disponer de este dato en un paciente.

Tabla 4. Edad con respecto a la técnica elegida

\begin{tabular}{|c|c|c|c|}
\hline TRS & N & MEDIA DE EDAD & DE \\
\hline HD & 28 & 67,36 & 12,91 \\
\hline DP & 9 & 47,56 & 13,50 \\
\hline TX VIV0 & 2 & 43 & 21,21 \\
\hline TTO CONSERV & 6 & 82,83 & 5,70 \\
\hline
\end{tabular}

Al analizar la edad respecto al tipo de técnica en la que comienza el paciente se han hallado diferencias estadísticamente significativas $(p=0,003)$. Estas diferencias se han hallado entre HD vs DP $(p=0.170)$ con una diferencia de medias de 17,3 años (IC95\%: $-4,8-39,4)$ mayor para HD. Entre DP vs tto. Conservador $(p<0,03)$ también se han mostrado diferencias.

Se han encontrado diferencias estadísticamente significativas entre elección y el tipo de actividad laboral $(p=0,006)$ siendo para los jubilados el de primera elección la HD (18/45 [40\%]) seguido del tratamiento conservador con un $13 \%(6 / 45)$.

Al analizar la relación entre actividad laboral frente a elección y sexo, se han observado que para los hombres existe una asociación entre estas dos variables ( $p=0,029)$; en cambio, para las mujeres no se ha mostrado tal asociación $(p=0,131)$. Es decir, hay más hombres jubilados que eligen HD que DP y más hombres activos que eligen DP. Esto no se muestra en el grupo de mujeres.

Tabla 5. Actividad laboral con respecto a la técnica elegida

\begin{tabular}{|c|c|c|c|c|}
\hline $\begin{array}{c}\text { ACTIVIDAD } \\
\text { LABORAL }\end{array}$ & HD & DP & $\begin{array}{c}\text { TX } \\
\text { VIVO }\end{array}$ & $\begin{array}{c}\text { TTO. } \\
\text { CONSERV }\end{array}$ \\
\hline JUBILAD0 & $\begin{array}{c}18 \\
(40 \%)^{*}\end{array}$ & $\begin{array}{c}2 \\
(4,4 \%)\end{array}$ & $\begin{array}{c}0 \\
(0 \%)\end{array}$ & $\begin{array}{c}6 \\
(13,3 \%)\end{array}$ \\
\hline ACTIV0 & $\begin{array}{c}3 \\
(6,7 \%)\end{array}$ & $\begin{array}{c}4 \\
(8,9 \%)\end{array}$ & $\begin{array}{c}1 \\
(2,2 \%)\end{array}$ & $\begin{array}{c}0 \\
(0 \%)\end{array}$ \\
\hline DESEMPLEADO & $\begin{array}{c}7 \\
(15,6 \%)\end{array}$ & $\begin{array}{c}2 \\
(4,4 \%)\end{array}$ & $0(0 \%)$ & $\begin{array}{c}0 \\
(0 \%)\end{array}$ \\
\hline BAJA & $\begin{array}{c}0 \\
(0 \%)\end{array}$ & $\begin{array}{c}1 \\
(2,2 \%)\end{array}$ & $\begin{array}{c}1 \\
(2,2 \%)\end{array}$ & $\begin{array}{c}0 \\
(0 \%)\end{array}$ \\
\hline
\end{tabular}

No se ha mostrado que exista una asociación entre el sexo y la elección $(p=0,673)$. Es decir, que entre hombres y mujeres no hay diferencias a la hora de elegir técnica.

\section{Conclusiones:}

- En nuestra muestra observamos que todavía hay un alto porcentaje de pacientes que comienzan TRS de forma aguda (33\%). 
- Hay una elevada tasa de analfabetismo (10,9\%), siendo mayor en mujeres.

- La elección de DP como tratamiento renal sustitutivo en nuestra población está condicionada por problemas socio-económicos.

- Un número significativo de pacientes elige tratamiento conservador asistido en domicilio (15,2\%).

- La elección de TRS está condicionada por la edad, como era de esperar, los pacientes que eligen DP son más jóvenes que los que eligen HD o tratamiento conservador.

- La elección del trasplante de vivo en prediálisis comienza a ser una realidad.

Recibido: 10 Febrero 2012

Revisado: 10 Marzo 2012

Modificado: 5 Septiembre 2012

Aceptado: 10 septiembre 2012
6. Bardón Otero E, Marti i Monros A, Vila Paz ML. Enfermería en la consulta de enfermedad renal crónica avanzada (ERCA). Nefrología: Vol. 28. Suplemento 3. 2008: 53-56.

\section{Bibliografía}

1. Pastor J. L, Julián J.C. Claves del proceso de información y elección de modalidad de diálisis en pacientes con insuficiencia renal crónica. Nefrología: Volumen 1.Suplemento 1. 20105: 15-20.

2. BOE. Ley $41 / 2002$ de 14 de noviembre. 274 : 4012640133.

3. Selgas R, Aguilar J, Julián J.C, Toledo A. Realidad y futuro en el tratamiento de la enfermedad renal crónica avanzada. Nefrología: Volumen 27. Suplemento 6. 2007: 689-693.

4. Sánchez Tomero JA. Planificación anticipada e inicio de diálisis. Nefrología: Vol. 29. Suplemento 4. 2009: 285-287.

5. Górriz JL, Otero A. Impacto socio sanitario de la enfermedad renal crónica avanzada. Nefrología: Vol. 28. Suplemento 3. 2008: 7-15. 